The search for black hole binaries using a genetic algorithm

This article has been downloaded from IOPscience. Please scroll down to see the full text article.

2009 Class. Quantum Grav. 26204011

(http://iopscience.iop.org/0264-9381/26/20/204011)

View the table of contents for this issue, or go to the journal homepage for more

Download details:

IP Address: 194.94.224.254

The article was downloaded on 11/06/2010 at 10:09

Please note that terms and conditions apply. 


\title{
The search for black hole binaries using a genetic algorithm
}

\author{
Antoine Petiteau, Yu Shang and Stanislav Babak \\ Max-Planck-Institut fuer Gravitationsphysik, Albert-Einstein-Institut, Am Muchlenberg 1, \\ D-14476 Golm bei Potsdam, Germany \\ E-mail: Antoine.Petiteau@aei.mpg.de
}

Received 9 May 2009, in final form 24 July 2009

Published 6 October 2009

Online at stacks.iop.org/CQG/26/204011

\begin{abstract}
In this work, we use a genetic algorithm to search for the gravitational wave signal from the inspiralling massive black hole binaries in the simulated Laser Interferometer Space Antenna (LISA) data. We consider a single signal in the Gaussian instrumental noise. This is a first step in preparation for analysis of the third round of the mock LISA data challenge. We have extended a genetic algorithm utilizing the properties of the signal and the detector response function. The performance of this method is comparable, if not better, to already existing algorithms.
\end{abstract}

PACS numbers: $04.30 . \mathrm{Tv}, 04.80 . \mathrm{Nn}$

(Some figures in this article are in colour only in the electronic version)

\section{Introduction}

Coalescence of two massive black holes (MBHs) in the galactic nuclei is the strongest and most promising source of gravitational waves (GW) for Laser Interferometer Space Antenna (LISA). This source is so powerful that the merger will be detected throughout the Universe. The observed event rate and the recovered parameters of the binary systems will allow us to trace the history of $\mathrm{MBH}$ formation [1]. Gravitational wave observations should enable us to measure masses and spins of MBHs in the binary with unprecedented accuracy [2]. It is expected that almost all the MBHs are spinning, and the value of spin might vary according to the channel of MBH formation. For example, if a significant part of the mass of a MBH was acquired through an accretion disk then one can expect the spin of the MBH close to the maximum possible value.

The search for inspiralling binaries in LIGO/VIRGO data usually utilizes templates for non-spinning compact objects. The reason for such a choice is that non-spinning templates can achieve a reasonable match which is sufficient for the detection [3]. The follow-up analysis could reveal whether compact objects have spins. In the case of LISA, one usually does not 
look for a detection but rather for an accurate estimation of parameters. As it was shown in $[2,4]$, including spins should significantly improve the estimation of parameters due to the de-correlation of the parameters entering the expression of the GW phase.

Several algorithms for detecting non-spinning $\mathrm{MBH}$ binaries in simulated LISA data have already been demonstrated [5-8]. In this paper, we describe a particular adaptation of a genetic algorithm to search for the GW signal from the inspiral of two MBHs. Genetic algorithms (GA) belong to the family of optimization methods, i.e. they look for extrema. The first application of GA in LISA data analysis was proposed in [9] for galactic binaries. The general principle is that a waveform template is associated with an organism, and parameters play the role of the set of genes defining this organism. The logarithm of likelihood obtained with a given template defines the quality of the organism. Then we evolve a set (colony) of organisms by using breeding, mutation and custom-designed accelerators with the aim of finding the genotype with the highest quality. This corresponds to the standard Darwin's principle: 'weak dies, strong lives', or translated into the conventional data analysis language: by evolving a set of templates, we are searching the parameters that maximize the likelihood.

The work presented below is a first step toward the analysis of the third round of mock LISA data challenge. The mock data set consists of the Gaussian instrumental noise, galactic background and four to six signals from the inspiralling spinning $\mathrm{MBH}$ binaries in a quasicircular orbit [11]. We made a number of simplifications: (i) we consider a single signal in the Gaussian instrumental noise and (ii) we fix all the parameters of the signal except masses, sky location and time of coalescence. Effectively we have reduced the problem to the non-spinning case; however, the signal is quite different from the non-spinning one. In fact, we did not put spins equal to zero, we have fixed them; therefore, the amplitude and phase modulations caused by the presence of spins are still there. The GA comes with a large number of options and many free parameters, and the above simplifications allow us to concentrate ourselves on tuning the algorithm before extending it to more complicated data and signal. In addition, this enables us to estimate performance of the algorithm and to compare it with the alternative approaches.

The structure of the paper is as follows. We describe the model of the GW signal in section 2. Then we give the basic principles of a standard GA in section 3. In section 4, we consider some specific modification and improvements of GA suitable for MBH binaries. We give and discuss our results in section 5, and we conclude with the summary in section 6 .

\section{Template and quality estimation}

\subsection{Model of the signal}

The waveform is described by 15 parameters: the two masses $m_{1}$ and $m_{2}$, the initial direction of the orbital angular momentum, polar angle $\theta_{L}$ and azimuthal angle $\phi_{L}$, the initial direction of spins, polar angles $\theta_{S_{1}}$ and $\theta_{S_{2}}$ and azimuthal angles $\phi_{S_{1}}$ and $\phi_{S_{2}}$, the dimensionless spin parameters, $\chi_{1}$ and $\chi_{2}$, the time at coalescence $t_{c}$, the phase at coalescence $\Phi_{c}$, the ecliptic coordinates of the source latitude $\beta$ and longitude $\lambda$, and the luminosity distance $D_{L}$.

The spin-orbit and the spin-spin interactions induce a precession of each binary member's spin vector and the orbital angular momentum. The precession equations are given in [10] and the waveform used in the mock data is described in detail in [11].

We have used two orthogonal (with uncorrelated noise) TDI (time delay interferometry) channels $\mathrm{A}$ and $\mathrm{E}$ in the phase domain (strain). TDIs are the time delayed combinations of the measurements which significantly reduces the laser noise [12, 13]. In our template, we consider a long wavelength approximation of these signals $[14,15]$. This approximation 
( $L \omega \ll 1$, where $L$ is the armlength of LISA and $\omega$ is an instantaneous frequency of GW) works pretty well below approximately $5 \mathrm{mHz}$. We have also assumed rigid LISA with equal arms. Then the waveform takes the following form $[14,15]$ :

$$
\begin{gathered}
h_{I}(t) \simeq 2 L \sin \Delta \phi_{2 L}\left(t_{k}\right)\left\{-h_{S 0+}\left(t_{k}\right)\left[\cos \left(2 \psi\left(t_{k}\right)\right) F_{+I}\left(t_{k}\right)-\sin \left(2 \psi\left(t_{k}\right)\right) F_{\times I}\left(t_{k}\right)\right] \sin \phi^{\prime}\left(t_{k}\right)\right. \\
\left.+h_{S 0 \times}\left(t_{k}\right)\left[\sin \left(2 \psi\left(t_{k}\right)\right) F_{+I}\left(t_{k}\right)+\cos \left(2 \psi\left(t_{k}\right)\right) F_{\times I}\left(t_{k}\right)\right] \cos \phi^{\prime}\left(t_{k}\right)\right\},
\end{gathered}
$$

where $I=\{A, E\}, t_{k}$ is the time in the LISA frame, $\psi$ is the polarization angle, $h_{S 0+}$ and $h_{S 0 \times}$ are the polarization amplitudes of the $\mathrm{GW}$ in the source reference frame, $\Delta \phi_{2 L}(t)=(\phi(t)-\phi(t-2 L)) / 2, \phi^{\prime}(t)=(\phi(t)+\phi(t-2 L)) / 2$ with $\phi(t)$ being the phase of GW. The antenna pattern functions $F_{+I}$ and $F_{\times I}$ have the following expression:

$$
\begin{aligned}
F_{+}\left(\theta_{d}, \lambda_{d} ; t, \Omega\right)= & \frac{1}{32}\left[6 \sin \left(2 \theta_{d}\right)\left(3 \sin \left(\Phi_{T}(t)+\lambda_{d}+\Omega\right)-\sin \left(3 \Phi_{T}(t)-\lambda_{d}+\Omega\right)\right)\right. \\
& -18 \sqrt{3} \sin ^{2} \theta_{d} \sin \left(2 \Phi_{T}(t)+\Omega\right)-\sqrt{3}\left(1+\cos ^{2} \theta_{d}\right) \\
& \left.\times\left(\sin \left(4 \Phi_{T}(t)-2 \lambda_{d}+\Omega\right)+9 \sin \left(2 \lambda_{d}+\Omega\right)\right)\right] \\
F_{\times}\left(\theta_{d}, \lambda_{d} ; t, \Omega\right)= & \frac{1}{16}\left[\sqrt{3} \cos \theta_{d}\left(\cos \left(4 \Phi_{T}(t)-2 \lambda_{d}+\Omega\right)-9 \cos \left(2 \lambda_{d}+\Omega\right)\right)\right. \\
& +6 \sin \theta_{d}\left(\cos \left(3 \Phi_{T}(t)-\lambda_{d}+\Omega\right)\right. \\
& \left.\left.+3 \cos \left(\Phi_{T}(t)+\lambda_{d}+\Omega\right)\right)\right]
\end{aligned}
$$

with $\theta_{d}=\beta+\pi / 2, \lambda_{d}=\lambda+\pi, \Phi_{T}(t)=2 \pi t /$ year and $\Omega=0$ for $\mathrm{A}$ and $\Omega=\pi / 4$ for $\mathrm{E}$.

\subsection{Quality estimation}

As we have mentioned before, we associate the quality of the organism with the logarithm of the likelihood. For a template $h(\widehat{\theta})$ the likelihood is given by

$$
\mathcal{L}(\widehat{\theta})=C \mathrm{e}^{-\langle s-h(\widehat{\theta}) \mid s-h(\widehat{\theta})\rangle / 2},
$$

where $s$ is the signal and $\langle h \mid s\rangle$ is the inner product defined as

$$
\langle h \mid s\rangle=2 \int_{0}^{\infty} \mathrm{d} f \frac{\tilde{h}(f) \tilde{s}^{*}(f)+\tilde{h}^{*}(f) \tilde{s}(f)}{S_{n}(f) .}
$$

The tilde denotes the Fourier transform and $S_{n}(f)$ corresponds to the power spectral density of LISA instrumental noise (plus possible galactic background approximated as described in [16]). The set of parameters $\widehat{\theta}$ maximizing the likelihood is called the maximum likelihood estimator [17-19], and that is what we are searching for.

Before using some numerical maximization methods, it is possible to maximize over the luminosity distance and the phase at coalescence analytically. This is the usual procedure routinely used in the ground-based GW data analysis (see for example [20]).

We also efficiently maximize the likelihood over the time of coalescence by sliding the template across the data (we use correlation instead of the inner product). This procedure is described in $[6,21]$.

\section{Genetic algorithm}

\subsection{Basic principle}

We start with a brief introduction to the genetic algorithm, then give a detailed implementation and describe specific modifications to solve the problems at hand. 
The GA is based on the natural selection principle which is the evolution theory established by $\mathrm{C}$ Darwin. In the nature, the organisms adapt themselves to their environment: the smartest/strongest/healthiest organism has most chances to survive and participate in breeding to produce the offspring. These two processes, selection and breeding, are used in the genetic algorithm for making a new generation. Since the best organisms are most likely to participate in breeding, the new generation should be better than the previous one (at least no worse). In order to increase the exploration of the possible organisms, a third process, called mutation, has been introduced. One needs to associate some measure of 'goodness' with each organism. In our case, it is the logarithm of the likelihood $(\log \mathcal{L})$, which we want to 'improve' through the evolution of the organisms.

Each GW template is associated with an organism. And the set of parameters characterizing the signal, $\widehat{\theta}$, play the role of genes. For describing genes, we use a binary representation of each parameter. The allowed resolution for each parameter depends on the number of bits used for describing it. If the parameter $x_{k}$ has a prior range between $x_{k, \min }$ and $x_{k, \max }$, its resolution is $\Delta x_{k}=\left(x_{k, \max }-x_{k, \min }\right) / 2^{N_{k}}$, where $N_{k}$ is the number of bits.

We keep the number of organisms in each generation fixed and allow only one active generation (one generation at the time): it means that after the breeding process all the parents are replaced by the children. Next we describe in detail the three main processes: selection, breeding and mutation.

\subsection{Selection}

The selection process chooses the parents for breeding. The probability of selecting one organism is related to its quality in the following way. First the quality $Q_{i}$, i.e. the maximized log-likelihood, of all the organisms is computed (index $i$ refers to the organism). Then we attach to each organism the probability to be chosen for breeding as $Q_{i} / \sum_{j}^{N} Q_{j}$ and apply the roulette selection method. With this method, the 'good' organisms are chosen more often than the 'bad' ones. Here we do not take into account the 'geographical' proximity between parents (in other words, possible correlation between templates in the same generation). By forbidding the breeding between correlated parents, we might improve the efficiency to explore larger parameter space, but we reduce the overall resolution of the method, so our selection is based only on the quality.

\subsection{Breeding}

The second process in GA is breeding. Two selected parents produce one child. The genes of the child are constructed by mixing the corresponding genes of each parent. We take one part from the first parent and the other part from the second parent. Depending on which parts are chosen, there are several types of breeding. We use two different types: cross-over one point (mid-point) and cross-over two points. For the cross-over one point, each gene of the child is combined out of the first $N / 2$ bits of the first parent and last $N / 2$ bits of the second parent (see the left panel of figure 1). It is similar to cross-over two points: the gene of the child is built from three equal parental parts (see the right panel of figure 1).

The first generation is chosen randomly by drawing parameters uniformly within the priors specified in [11]. The chosen selection implies that the quality of our organisms more likely will increase with each generation. But if we use only breeding, the range of resulting genes is quite restricted: it is just a combination of the parts from the initial generation. Therefore, the exploration of the parameter space is rather poor. 


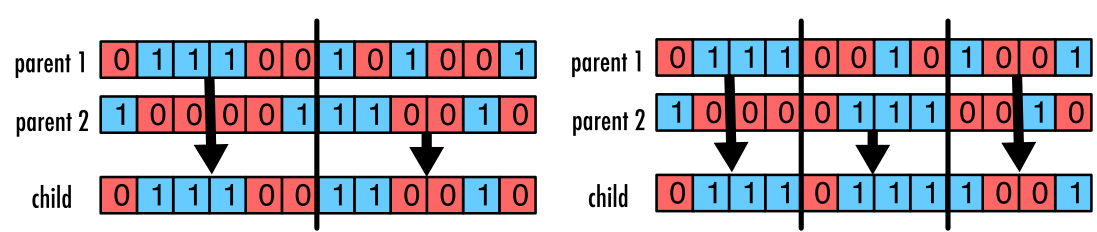

Figure 1. Examples of breeding: cross-over one point on the left and cross-over two points on the right panels.

\subsection{Mutation}

In order to increase the exploration of the parameter space, we need to introduce mutation. It works in a way similar to the mutation in the nature. Mutation is a random change of a few alleles in a gene; in our algorithm, it corresponds to changing a few bits in a gene of an organism. The probability of mutation is described by a number from 0 to 1 and it is called the probability mutation rate (PMR). We mutate each gene independently and there are several types of mutation. First we need to decide whether we mutate a gene or not and, if yes, we need to decide on the rule of mutation (how we do it). The first possibility: we always mutate the gene and mutation is applied to each bit of gene independently. For each bit, we draw a random number from 0 to 1 and flip the bit if the number is lower than PMR. The second possibility is that we decide to mutate the gene if a randomly drawn number is below PMR. In this case, we have used two different rules for mutation: (i) we flip $N$ randomly chosen bits and (ii) we flip $N$ adjacent bits. Different types of mutation together with the value of PMR define the exploration area of the parameter space. Large PMR implies frequent mutations and therefore exploration of large parameter space; this is a desired feature in the beginning of the search.

\section{Accelerations of the genetic algorithm}

We have introduced above three fundamental processes of any GA. However, one might need to evolve a very large number of generations before finding the best organisms. To reduce the required number of generations and give more stability to the algorithm, we introduce several accelerators which are used in our search and described below.

\subsection{Standard accelerators}

In this part, we describe the known accelerators often used in GA.

4.1.1. Elitism. The elitism (or cloning) implies that we keep the best organism through the generations. It is possible to clone one or several best organisms into the new generation. The elitism stabilizes the GA because the best point in the parameter space is always preserved and it guarantees the convergence of the algorithm.

4.1.2. Simulated annealing. The simulated annealing was already employed in LISA data analysis [6] and proven to be very useful. The smoothness of the quality surface is controlled by the introduced temperature. If it is hot, the quality surface is very smooth and nearly all the organisms ('good' and 'bad') can be selected for breeding with a similar probability. If it is cold, the quality surface is very peaked around the maxima and only the best organisms can be selected. Usually, we use a high temperature at the beginning of the search to increase the exploration area and cool it down as we approach the solution. 
4.1.3. Evolution of PMR. As we mentioned above, the PMR controls the exploration of the parameter space. Therefore, we can control the search area by changing the PMR during the evolution. Usually we start with a large value for the PMR (about 0.2 ), then slowly decrease it and give more importance to the breeding. Note that we can control the exploration area using both simulated annealing and PMR. We use them both as they perform somewhat differently (simulated annealing uses combination of the initial genes without adding new) and the best result is usually achieved if we combine them together.

\subsection{Others accelerations}

In this part, we describe non-standard acceleration processes introduced by us and which utilize the properties of the signal and/or the antenna beam pattern.

4.2.1. Brother. We introduced what we call 'the brother' system. With each clone, we associate one organism (brother) created by using specific rules. In our application of the GA for black hole binaries, the brother is used for searching around the mirrored (antipodal) sky position of each clone (we keep all the parameters of the clone but flip the sky location).

4.2.2. Local mutation. The binary representation creates boundaries in the parameter space. For example, the separation between the gene value 011111 and 100000 is equal to the resolution $\Delta x$ (i.e. minimal distance), but, as one can see, it is necessary to flip all the bits for making this small step. This problem can be solved by introducing the local mutation. It corresponds to a small (of order few $\Delta x$ ) random change in the parameter value after mutation which can push it across the boundary.

4.2.3. Fixing bits. To find the traces of the signal in the data, we want to explore a large parameter space at the beginning of the search. But after few hundred generations, some of the parameters are already quite well estimated so the exploration of the large range in those parameters starts to slow down the search. At this stage, we fix (freeze) the most significant bits of those parameters which reduces the allowed dynamical range.

4.2.4. Specific breeding and mutation. As mentioned above, different types of breeding and mutation have different properties (main difference is in the exploration area around the best organism). The genes (i.e. parameters) do not have the same evolution during the search. For example, the time of coalescence and the chirp mass converge to the answer quicker than other parameters. We can customize our algorithm so that each gene has its own type of breeding and mutation and which can also evolve together with the colony of organisms.

4.2.5. Change of environment. A change of the environment corresponds to a change in the quality distribution in the parameter space. The main idea here is that the 'good' organism remains 'good' in the different environment. Here, we change the maximum allowed frequency of our template (terminate the inspiral at earlier times). The quality distribution for the shortened waveform does not have the same local maxima as the quality obtained with the full waveform, but the same global maximum. Therefore, the alternating use of full and chopped waveforms helps to move the search away from the local maxima where it has a tendency to get stack. 

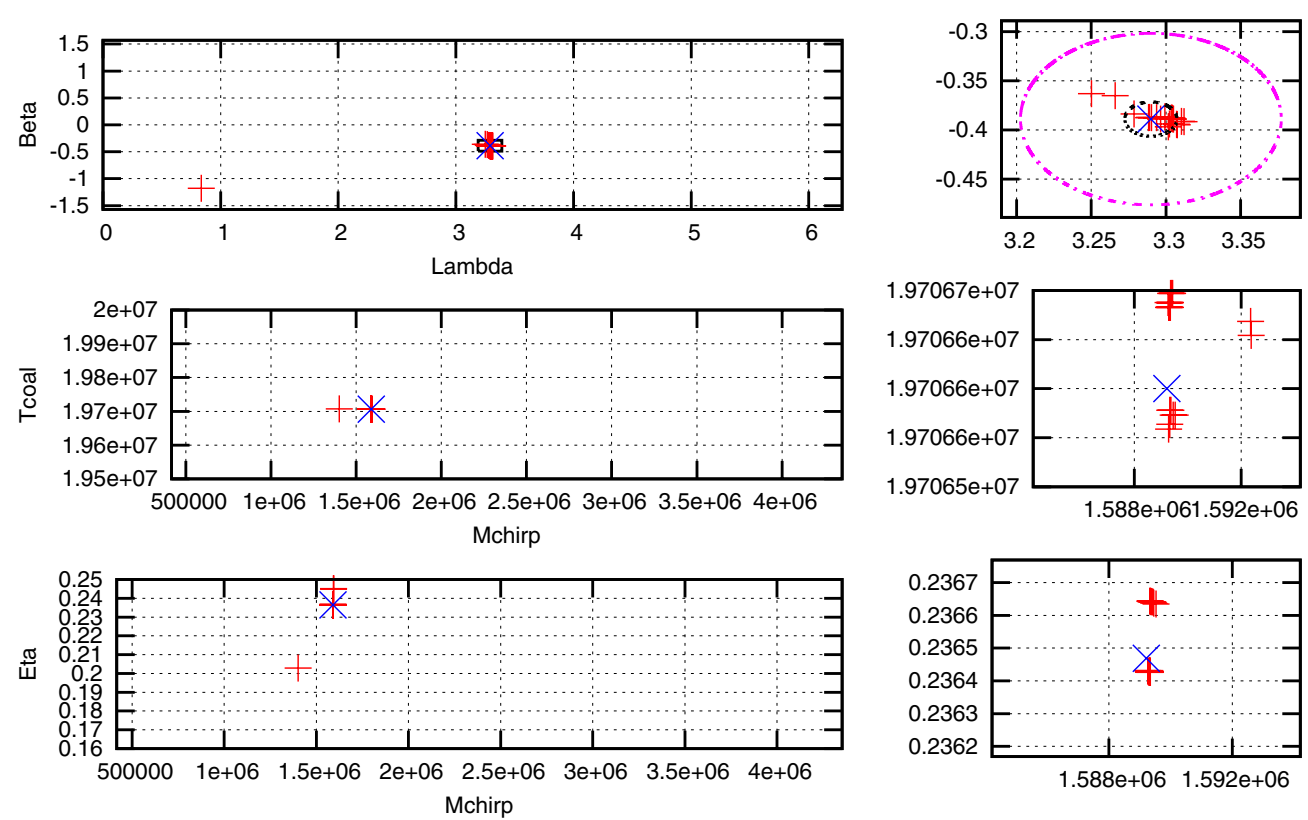

Figure 2. Distribution of 50 best organisms from 50 runs of GA. The source coalesces within the observational time. The pluses ' + ' correspond to the best organism of each run after 2000 generations. The cross ' $x$ ' corresponds to the true value of the parameters. On the left side, graphs show the full prior range of parameters. On the right side, we zoom onto the area around the true value. On the top-right graph, the big circle corresponds to the error of 19.63 square degrees and the small one is 0.785 square degree error area.

\section{Results}

Our ultimate intent is to use GA in the Mock LISA Data Challenge 3 [11]. The mock data consist of the Gaussian stationary instrumental noise, a galactic cyclo-stationary background and four to six signals from the inspiralling spinning BHs in the quasi-circular orbits.

As we have mentioned in the introduction, we have simplified the search with the prime purpose to explore the numerous options which GA provide and make the first assessment of the GA performance. We consider a single GW signal in the instrumental noise. Further we have fixed all the parameters to their true values besides masses, sky location and time of coalescence. The spin terms come into the GW phase at 1.5 post-Newtonian (spinorbital coupling) orders and at second post-Newtonian (spin-spin) orders, so they bring rather small corrections to the phase. In our simplification, we want to make sure that we can find spin unrelated parameters before hunting the small corrections. We do not exclude the possibility that including all the 15 parameters in the search will bring more correlations between parameters and creating more local maxima in the likelihood which will require extension of our algorithm.

Let us emphasize that the main aim of this paper is to describe the application of GA to the search for SMBH binaries in the LISA data, tune the algorithm by using the simplified data and reduced parameter space and assess the performance of the algorithm. We do not intend to conduct neither an intensive study of the performance nor the detailed comparison with other algorithms. This simplified search is a first step toward the search for precessing SMBH binaries. Application of GA to the full problem will be described in the separate publication [22]. 

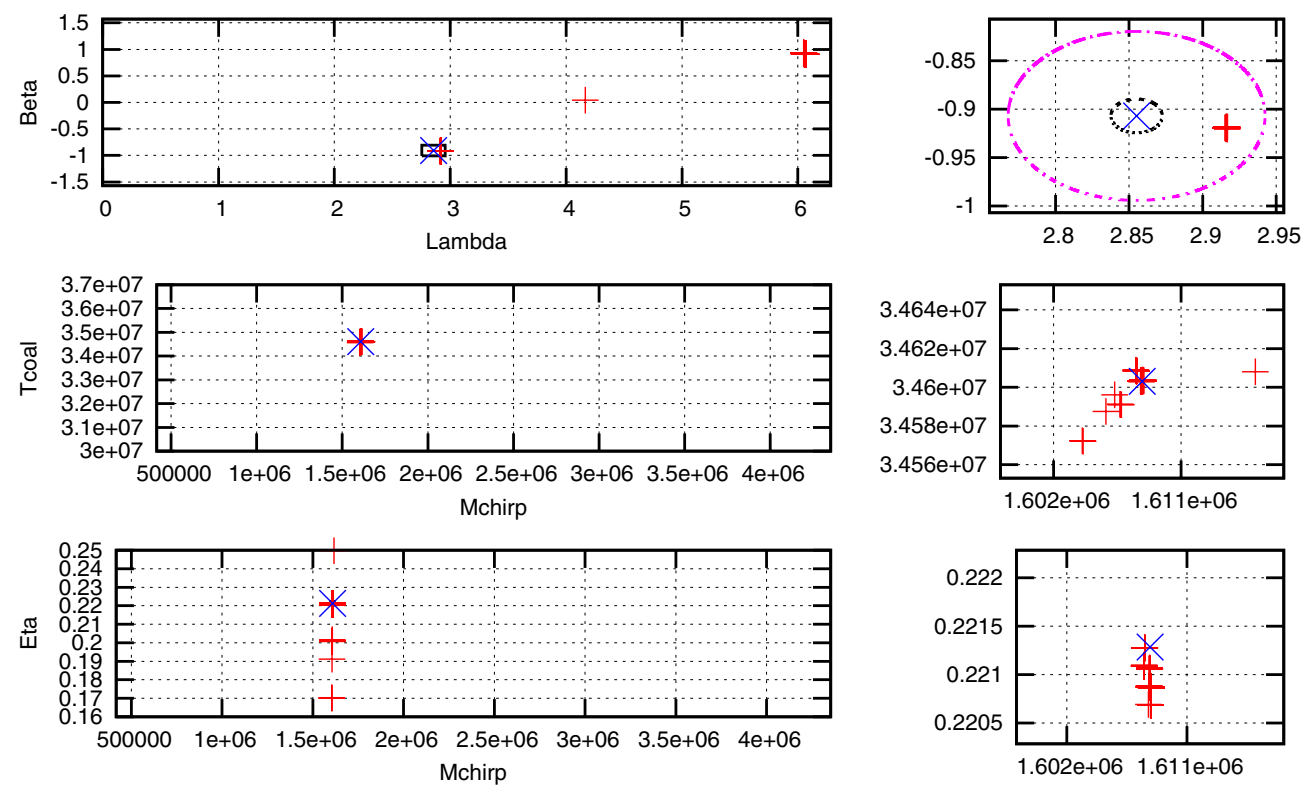

Figure 3. Results of 50 runs of GA for the source with coalescence beyond the end of the observational time. The pluses ' + ' correspond to the best organisms of each run after 2000 generations. The cross ' $x$ ' corresponds to the true value of the parameters. On the left side, graphs show the full prior range of the parameters. On the right side, we zoom around the true value. On the top-right plot, the big circle corresponds to the 19.63 square degrees error circle and the small one is for the 0.785 square degree error region.

The sources can be separated into two types: the sources with a time of coalescence within the observational time and the ones which coalesce outside the observational time. We have investigated those two cases separately and we present our results below.

\subsection{Coalescence within the observational time}

We have analyzed 1 year of simulated LISA data including the signal with following parameters: signal-to-noise ratio $\mathrm{SNR}=1250(\beta=-0.38896 \mathrm{rad}, \lambda=3.28992 \mathrm{rad}$, $\left.t_{c}=19706568.3273 \mathrm{~s}, M_{c}=1589213.34 M_{\odot}, \eta=0.23647\right)$. We have run GA 50 times with different random initial states and for 2000 generations each (usually enough to get to the answer). Figure 2 shows the best organism obtained from each evolution. For 48 out of 50 runs, the median absolute error in the sky position is 0.12 square degrees, the median fractional error in the chirp mass is $0.0077 \%$, the median error in $\eta$ is $0.045 \%$ and the median absolute error in time of coalescence is $114 \mathrm{~s}$. Note that the errors (especially for the time of coalescence) are larger than what would be predicted by the Fisher matrix analysis. This is due to a large systematic error caused by using the long wavelength approximation. This systematic error is removed if we employ the rigid adiabatic approximation or the full response. The main result here is that we have found the global maximum.

\subsection{Coalescence outside the observational time}

Similarly we have considered 1 year of simulated LISA data with a source coalescing outside the time of observation. The SNR of that signal was 47, and other parameters are $\left(\beta=-0.90706300796 \mathrm{rad}, \lambda=2.85511464611 \mathrm{rad}, t_{c}=34603008.0 \mathrm{~s}\right.$, 
$\left.M_{c}=1608239.35302 M_{\text {solar }}, \eta=0.22129\right)$. Figure 3 shows 50 best organisms from each run of GA.

The results are slightly worse, mainly, because of the lower SNR: for 22 out the 50 jobs, the median absolute error on the sky is 2.4 square degrees, the median relative error on chirp mass is $0.0044 \%, \eta$ determined with the median error $0.18 \%$ and the absolute median error in the time of coalescence is $153 \mathrm{~s}$. Other 28 best organisms have found the opposite sky position (there is a very strong degeneracy for this low frequency source) with similar error in the chirp mass, $\eta$ and the time at coalescence. The systematic errors here are negligible (besides systematic bias in the sky location) and those results are consistent with an analytic analysis based on the Fisher information matrix.

\section{Conclusion}

We have presented an extended version of the GA to search for BHs in the simulated LISA data. We devised several acceleration procedures for GA which are based on the properties of the signal and the detector antenna pattern. The algorithm shows remarkable performance for a single signal in the simulated instrumental noise assuming that the precession-related parameters are known. The performance of GA is comparable to the best available algorithms based on Markov Chain Monte Carlo [11, 23]. We have recently applied GA to the full 15 parameters' search and obtained very good results which we delegate to the separate publication [22].

\section{Acknowledgments}

Work of AP and SB was supported in parts by DFG grant SFB/TR 7 'Gravitational Wave Astronomy' and by DLR (Deutsches Zentrum fur Luft- und Raumfahrt). YS was supported by MPG within the IMPRS program.

\section{References}

[1] Sesana A, Volonteri M and Haardt F 2007 Mon. Not. R. Astron. Soc. 3771711

[2] Lang R N and Hugues S A 2006 Phys. Rev. D 74122001

[3] Van Den Broeck C et al 2009 gr-qc/arXiv0904.1715

[4] Vecchio A 2004 Phys. Rev. D 70042001

[5] Brown D A, Crowder J, Cutler C, Mandel I and Vallisneri M 2007 Class. Quantum Grav. 24 S595

[6] Cornish N J and Porter E K 2006 Class. Quantum Grav. 245729

[7] Feroz F, Gair J R, Hobson M P and Porter E K 2009 gr-qc/arXiv0904.1544

[8] Gair J R and Porter E K 2009 gr-qc/arXiv0903.3733

[9] Crowder J, Cornish N J and Reddinger L 2006 Phys. Rev. D 73063011

[10] Apostolatos T A, Cutler C, Sussman G J and Thorne K S 1994 Phys. Rev. D 496274

[11] Babak S et al 2008 gr-qc/arXiv08062110

[12] Vallisneri M, Crowder J and Tinto M 2008 Class. Quantum Grav. 25065005

[13] Tinto M and Dhurandhar S V 2005 Living Rev. Rel. 84

[14] Cornish N J and Rubbo L J 2003 Phys. Rev. D 67022001

[15] Petiteau A 2008 PhD thesis Université Paris Diderot (Paris 7)

[16] Timpano S E, Rubbo L J and Cornish N J 2006 Phys. Rev. D 73122001

[17] Finn L S 1992 Phys. Rev. D 465236

[18] Cutler C and Flanagan E 1994 Phys. Rev. D 492658

[19] Owen B J 1996 Phys. Rev. D 536749

[20] Jaranowski P, Królak A and Schutz B F 1998 Phys. Rev. D 58063001

[21] Babak S 2008 Class. Quantum Grav. 25195011

[22] Petiteau A, Shang Y, Babak S and Feroz F 2009 in preparation

[23] Babak S et al 2008 Class. Quantum Grav. 25114037 